\title{
Molecular Mechanism for Lipid Flip-Flops
}

\author{
Andrey A. Gurtovenko*,† and Ilpo Vattulainen $\$, \S, \perp$ \\ Computational Laboratory, Institute of Pharmaceutical Innovation, University of Bradford, Bradford, West \\ Yorkshire, BD7 1DP, United Kingdom, Institute of Physics, Tampere University of Technology, P.O. Box 692, \\ FI-33101 Tampere, Finland, Helsinki University of Technology, P.O. Box 1100, FI-02015 HUT, Finland, and \\ MEMPHYS - Center for Biomembrane Physics, University of Southern Denmark, Odense, Denmark
}

Received: September 4, 2007

\begin{abstract}
Transmembrane lipid translocation (flip-flop) processes are involved in a variety of properties and functions of cell membranes, such as membrane asymmetry and programmed cell death. Yet, flip-flops are one of the least understood dynamical processes in membranes. In this work, we elucidate the molecular mechanism of pore-mediated transmembrane lipid translocation (flip-flop) acquired from extensive atomistic molecular dynamics simulations. On the basis of 50 successful flip-flop events resolved in atomic detail, we demonstrate that lipid flip-flops may spontaneously occur in protein-free phospholipid membranes under physiological conditions through transient water pores on a time scale of tens of nanoseconds. While the formation of a water pore is induced here by a transmembrane ion density gradient, the particular way by which the pore is formed is irrelevant for the reported flip-flop mechanism: the appearance of a transient pore (defect) in the membrane inevitably leads to diffusive translocation of lipids through the pore, which is driven by thermal fluctuations. Our findings strongly support the idea that the formation of membrane defects in terms of water pores is the rate-limiting step in the process of transmembrane lipid flip-flop, which, on average, requires several hours. The findings are consistent with available experimental and computational data and provide a view to interpret experimental observations. For example, the simulation results provide a molecular-level explanation in terms of pores for the experimentally observed fact that the exposure of lipid membranes to electric field pulses considerably reduces the time required for lipid flip-flops.
\end{abstract}

\section{Introduction}

Membranes of most animal cells are known to be asymmetric with regard to transmembrane distribution of lipids across a membrane. ${ }^{1,2}$ This asymmetry is crucial for an array of cellular functions and plays an important role, e.g., in membrane mechanical stability, ${ }^{3}$ modulation of the activity of membrane proteins, ${ }^{4}$ and programmed cell death. ${ }^{5}$ Therefore, to maintain an asymmetric transmembrane lipid distribution, cellular membranes have means to translocate (flip-flop) lipid molecules from one membrane leaflet to another.

One way cells make this happen is to employ special mechanisms to actively transport lipids across a lipid bilayer using specific membrane proteins, flippases, whose biological functions and relevance are widely recognized. ${ }^{6,7}$ To complement active translocation, cells also use passive transport mechanisms that facilitate the migration of lipids from one leaflet to another. For this purpose, the translocation may take place with the help of proteins or without them. ${ }^{8}$ The mechanisms associated with transmembrane transport in protein-free membranes are exceptionally poorly understood, however. It is commonly assumed that the transbilayer movement of lipids in protein-free membranes takes place as a single-molecule process, ${ }^{4,8}$ where the cooperative motion of nearby lipids allows the migrating lipid to more easily cross the transition state of the flip-flop event. ${ }^{9}$ However, since flip-flops are very rare

* Corresponding author. E-mail: A.Gurtovenko@bradford.ac.uk

$\dagger$ University of Bradford.

$\div$ Tampere University of Technology.

$\S$ Helsinki University of Technology.

$\perp$ University of Southern Denmark. processes, it has remained unclear whether this view is indeed the most plausible one. Yet the understanding of the molecular mechanisms of lipid flip-flops is of substantial biological importance since a loss of transmembrane lipid asymmetry can have severe consequences. ${ }^{10}$ For instance, while negatively charged phosphatidylserine lipids are usually located in the inner leaflet of the plasma membrane, their appearance in the outer leaflet is known to correlate with programmed cell death. ${ }^{5}$ In more general terms, understanding how membrane lipids maintain the asymmetric transmembrane distribution and achieve their nonrandom distribution in cells is one of the key challenges in cell biology. ${ }^{11}$

The reason lipid flip-flops and their detailed molecular mechanisms in the absence of flippases are so difficult to characterize is rather obvious. From an experimental point of view, it is a matter of resolution since these processes take place over molecular scales. Meanwhile, from a computational viewpoint, the atomistic modeling of flip-flops has been considered to be a prohibitive task for decades to come, since, in general, lipid flip-flops are very slow processes; they are characterized by an average life time from several hours to several days. ${ }^{12,13}$

However, there are strong indications that lipid translocation across a lipid membrane is a pore-mediated process. It has been demonstrated ${ }^{14}$ that the experimentally determined activation energy for radioactive chloride flux across lipid membranes is close to the activation energy of lipid flip-flop. ${ }^{12}$ Further, brief electric pulses (electroporation) have been shown to enhance the transbilayer mobility of phospholipids. ${ }^{15}$ All together, the above findings suggest that a major fraction of flip-flops takes place through water defects in lipid membranes. 
On the computational side, several observations of defectmediated lipid flip-flops have been reported; most of them were related to flip-flop events in lipid membranes far from physiological conditions, though. In particular, very recent lipid membrane simulations indicated that, when a lipid was dragged by an external force through the membrane interior, the formation of a small water pore was observed; the energies required for lipid flip-flop and for the formation of the pore were found to be identical. ${ }^{16}$ On the basis of these findings, the authors concluded that lipid flip-flop could be a defect-mediated process. Meanwhile, de Vries et al. have reported on the poremediated equilibration of lipids between two leaflets in the early phases of vesicle self-assembly $;{ }^{17}$ the results are appealing, but the far from equilibrium conditions under self-assembly are markedly different from conditions where the dynamics in stable cell membranes takes place. Later, a defect-mediated lipid flipflop was reported in molecular dynamics (MD) simulations of membranes under the influence of other external factors such as antimicrobial peptides ${ }^{18}$ and butanol. ${ }^{19}$ Finally, a recent computational study ${ }^{20}$ reported a single pore-mediated lipid flipflop that was driven by an extremely high transmembrane voltage, i.e., again under conditions far from those that can be observed in a cell. Furthermore, the authors observed that sodium cation flux can be a factor that facilitates the dragging of lipids across a water pore. Overall, despite their limitations (membrane exposure to external factors or nonequilibrium conditions), all the above studies support the view that flipflops in protein-free membranes may take place through defects in membranes.

In this paper, we show through extensive atomic-scale MD simulations that the computational approach can actually provide a great deal of insight into the mechanism (or one of the mechanisms) associated with lipid flip-flops. The simulations conducted under physiological conditions provide compelling evidence that, initially, the key event leading to flip-flop is the spontaneous formation of a water pore. Having formed, the water pore facilitates the spontaneous migration of lipids across the membrane (see Figure 1). On the basis of 50 flip-flop events resolved in atomic detail, we provide a detailed molecular picture for lipid translocation across a membrane and discuss the forces driving these processes. Remarkably, the average time required for a lipid to translocate through a preformed water pore was found to be around 60 ns. Together with the large number of flip-flops observed here, this strongly suggests that the actual flip-flop event is a rapid process, while the spontaneous formation of a water defect in lipid membranes is the ratelimiting step in the process of lipid flip-flop. The experimental data that is available for comparison and discussed at the end of this article is consistent with the simulation results.

The most notable contribution of this work is the unprecedented view for a large number of diffusive (driven by thermal fluctuations) lipid flip-flops observed in atomistic detail, thus clarifying the mechanism of flip-flops under conditions close to physiological ones. There is indeed ample reason to stress that, in the present study, the simulation conditions really match closely those observed in a cell close to equilibrium, without artificial external fields or far from equilibrium conditions.

\section{Methods}

The atomic-scale MD simulations were performed on lipid membranes comprised of zwitterionic dimyristoylphosphatidylcholine (DMPC) lipids. Force-field parameters for lipids were taken from the united atom force-field of Berger et al. ${ }^{21}$ the force-field has been shown to correctly reproduce the experi-
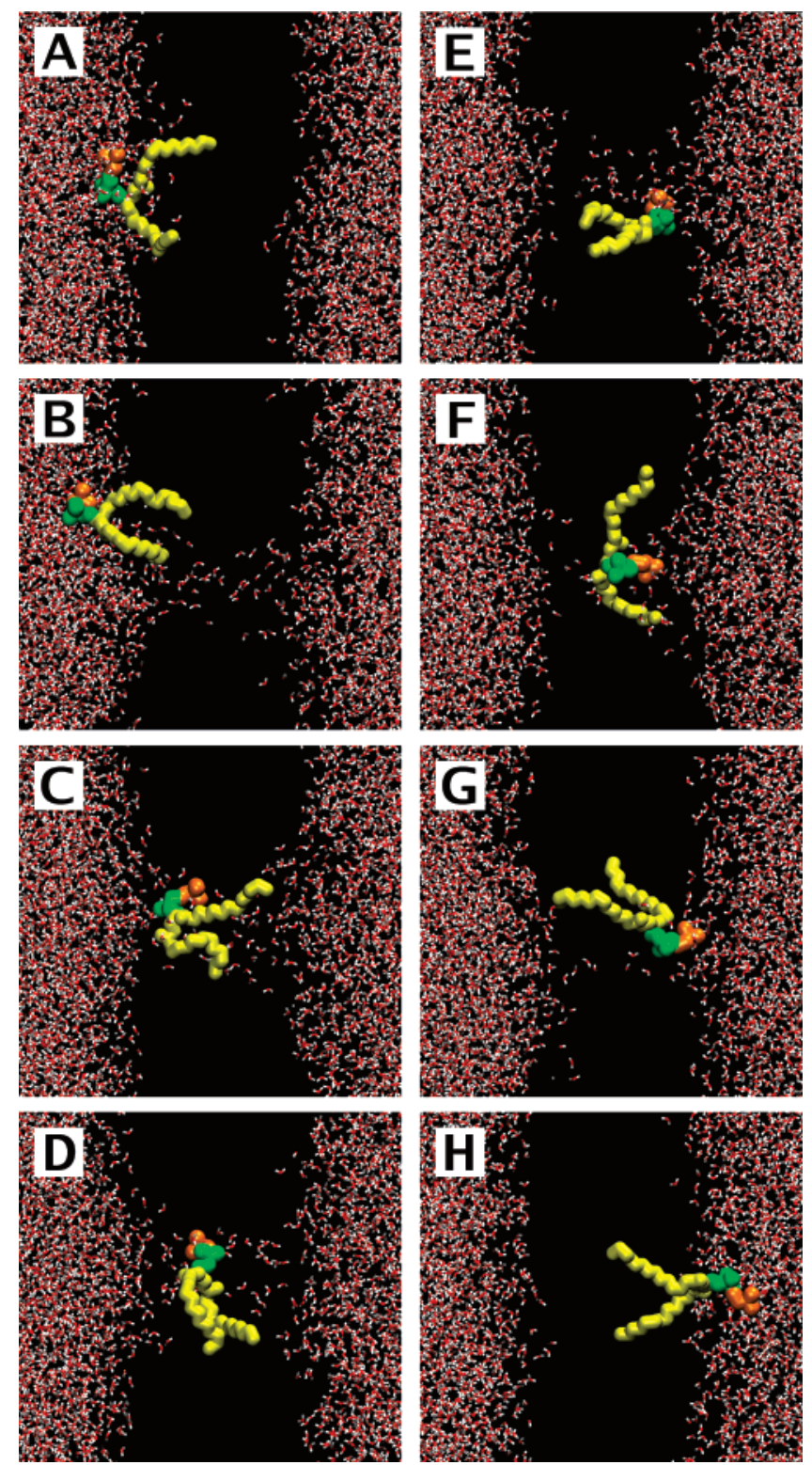

Figure 1. Pore-mediated lipid flip-flop: (A) 0 ps, (B) 43.85 ns, (C) $118.9 \mathrm{~ns}$, (D) $122.4 \mathrm{~ns}$, (E) $152.7 \mathrm{~ns}$, (F) $204.65 \mathrm{~ns}$, (G) $208.9 \mathrm{ns,} \mathrm{and}$ (H) 215 ns. Lipids (except for the flip-flopped one) are not shown; water is shown in red and white, acyl chains of the flip-flopped lipid are shown in yellow, and its choline and phosphate groups are shown in orange and green, respectively.

mentally measured area and volume per lipid (see, e.g., ref 22). Water was modeled using the simple point charge model. ${ }^{23}$ For sodium, potassium, and chloride ions, we employed the default set of parameters supplied within the GROMACS force field. ${ }^{24}$ The Lennard-Jones interactions were cut off at $1 \mathrm{~nm}$. For the electrostatic interactions, the particle-mesh Ewald method ${ }^{25,26}$ was used. The simulations were performed in the $N p T$ ensemble with temperature and pressure kept constant by the Berendsen scheme. ${ }^{27}$ Pressure was set to 1 bar. Temperature was set to $323 \mathrm{~K}$, which is well above the main phase transition temperature of a DMPC bilayer (297 K).

Water pores in lipid membranes were induced by adding salt $(\mathrm{NaCl}$ or $\mathrm{KCl}$ ) and by creating an imbalance of cations (sodium or potassium) across the membrane. ${ }^{22,28}$ To model the transmembrane ionic charge imbalance explicitly, a double-bilayer setup (i.e., two lipid bilayers of 128 lipids each in a simulation box) was employed, ${ }^{29,30}$ amounting to about 42000 atoms in the system. The time step used in the integration of equations 
TABLE 1: Summary of Lipid Flip-Flop Events

\begin{tabular}{ccrcc}
\hline system & salt $^{a}$ & $t_{\text {sim }}[\mathrm{ns}]^{b}$ & $N_{\text {flip-flop }}{ }^{c}$ & $t_{\text {flip-flop }}[\mathrm{ns}]^{d}$ \\
\hline 1 & $\mathrm{NaCl}$ & 215 & 8 & $66 \pm 13$ \\
2 & $\mathrm{NaCl}$ & 95 & 2 & $78 \pm 18$ \\
3 & $\mathrm{NaCl}$ & 125 & 5 & $78 \pm 9$ \\
4 & $\mathrm{NaCl}$ & 50 & 3 & $38 \pm 5$ \\
5 & $\mathrm{KCl}$ & $200^{e}$ & 10 & $70 \pm 10$ \\
6 & $\mathrm{KCl}$ & 35 & 5 & $27 \pm 3$ \\
7 & $\mathrm{KCl}$ & $200^{e}$ & 13 & $66 \pm 10$ \\
8 & $\mathrm{KCl}$ & 80 & 4 & $58 \pm 13$
\end{tabular}

${ }^{a}$ Type of salt used in a simulation. ${ }^{b}$ Total time of a simulation. Simulations were extended until a pore was closed, except for systems 5 and 7. ${ }^{c}$ Number of flip-flop events. ${ }^{d}$ Average duration of a lipid flip-flop process. ${ }^{e}$ Pore did not close over the course of the simulation.

of motion was 2 fs. In all, we considered eight different bilayer systems with pores; most systems were simulated until a pore was closed, with the exception of systems 5 and 7, which had pores open even after $200 \mathrm{~ns}$ (see Table 1). The total simulated time amounted to $1 \mu \mathrm{s}$. All simulations were performed using the GROMACS suite. ${ }^{24}$

\section{Results}

To study pore-mediated lipid flip-flops, water pores were first preformed in membranes composed of DMPC lipids. This was accomplished by creating a transmembrane imbalance of cations (either sodium or potassium ions), which induces a spatially and time-dependent electric field across the membrane. To this end, we employed a double-bilayer setup where the system included two lipid bilayers in the lamellar fluid phase. The ion concentration imbalance employed in this study was intentionally chosen to be rather large (six cations per bilayer of 128 lipids), so that the formation of water pores occurred on a nanosecond time scale. After a pore has been formed, one observes the transport of ions through the pore, which quickly discharges the transmembrane ionic charge imbalance and makes the pore metastable. A detailed discussion of the overall process of pore formation and subsequent ion leakage can be found elsewhere. ${ }^{22,28}$

There is reason to emphasize that the transmembrane leakage of ions is a much faster process compared to lipid flip-flop: the ionic charge imbalance across a membrane discharges almost fully within 5-6 ns after pore formation. Therefore, the leakage of ions can affect lipid flip-flops at a very early stage only. The remaining ionic charge imbalance after the first few nanoseconds of pore formation is essentially negligible (usually one cation per bilayer) or even zero, which corresponds to a complete discharging of the transmembrane potential. Water pores, being in a metastable state, stay open from about 35 to 200 ns.

After the formation of a water pore, we witness spontaneous pore-mediated translocation of lipid molecules from one leaflet to another. Table 1 summarizes the lipid flip-flop events observed. The overall process of lipid flip-flop is visualized in Figure 1 for one particular lipid of the simulation system 1 . Starting from an intact lipid membrane (Figure 1A), a water pore spanning the entire membrane is first formed; the pore is laterally located far away from the lipid in question and does not affect it (Figure 1B). After about 100 ns, the lipid diffuses laterally to the pore site and becomes part of the pore, lining the pore by its head group (Figure 1C). At this moment, the spontaneous diffusive translocation of a lipid through the pore initiates: it involves the progressive diffusion of a lipid head group in the pore coupled with the simultaneous desorption of lipid hydrocarbon chains out from the membrane leaflet (Figure
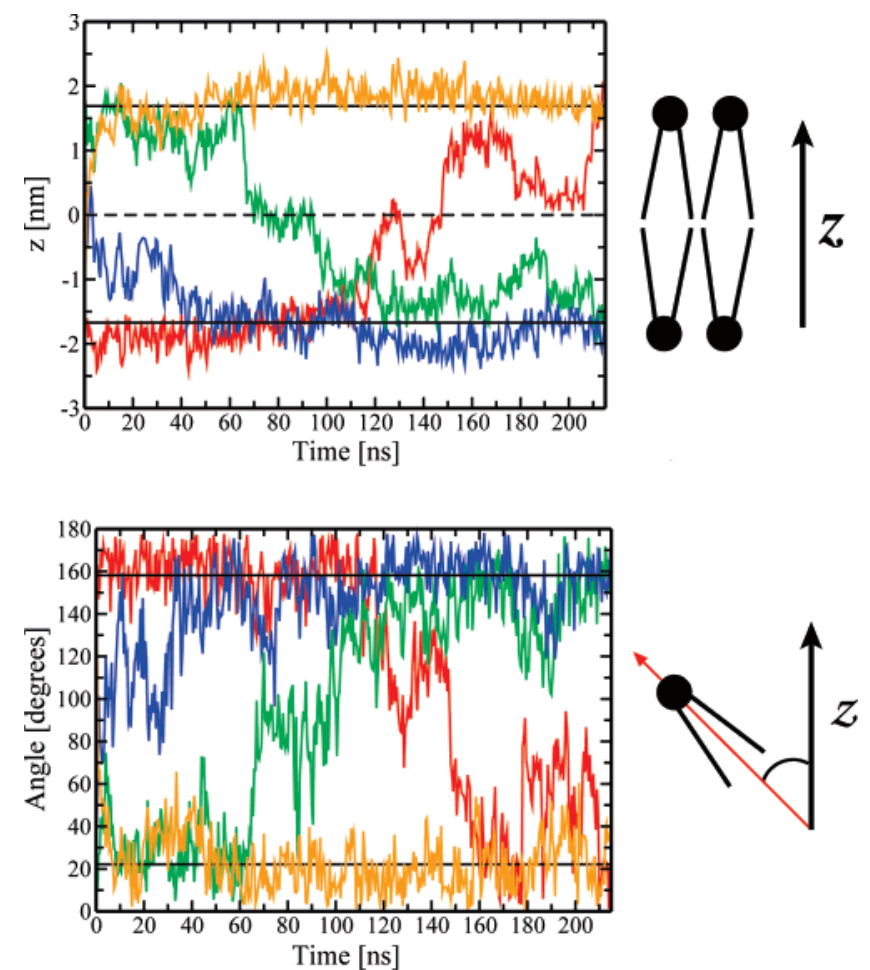

Figure 2. (Top) Time evolution of positions of the CMs of head groups for four flip-flopped lipids of system 1 . The $z=0$ corresponds to the center of the membrane; solid black lines show the average positions of lipid head groups in the two opposite leaflets, extracted from the intact membrane before pore formation. (Bottom) Time evolution of the tail-to-head orientation for the same four flip-flopped lipids. The tail-to-head orientation was characterized as the angle between the bilayer normal and the vector directed from the CMs of the acyl chains to the CMs of head groups. Solid black lines again show typical values of the angle for lipids in the opposite leaflets.

1D). This eventually leads to the appearance of the lipid in the opposite membrane leaflet, accompanied by the subsequent reorientation of the lipid (Figure 1E). The irreversible accommodation of a lipid in the opposite leaflet (if successful) turns out to be a rather slow process (more than $50 \mathrm{~ns}$ is required for the particular lipid considered here; see Figure $1 \mathrm{E}-\mathrm{G}$ ) since it involves spontaneous detachment of a head group out of pore "walls" and lateral diffusion of a lipid away from the pore. This, however, can be greatly facilitated by pore closure, which occurs at $t \simeq 210 \mathrm{~ns}$ for this particular system (Figure $1 \mathrm{H}$ ).

To further characterize lipid flip-flops, in Figure 2 we show the time evolution of the positions of centers of mass (CMs) of several lipid head groups. Also depicted in the same figure is the lipids' overall orientation within a membrane (shown here are the trajectories of four typical flip-flopped lipids of system 1). As seen, one can distinguish two somewhat different types of lipid flip-flops: (i) very fast flip-flops of lipids directly involved in the initial formation of a water pore (these are characterized by a rapid onset of translocation, which coincides with a pore formation event, and by a rather short time (10-20 ns) required for successful accommodation in the opposite leaflet (orange curve in Figure 2)); and (ii) flip-flops of lipids that are either involved in the initial pore formation but require considerably longer time to accomplish translocation (blue curve in Figure 2) or are initially remote from a pore but diffuse to the pore site with time (red and green curves; note that the red curve corresponds to the translocation of the lipid exemplified above in a series of snapshots shown in Figure 1). The number of flip-flops in category (i) is 9 out of 50 events observed (three 
for $\mathrm{NaCl}$ and six for $\mathrm{KCl}$ ), thus the majority of flip-flops (about $80 \%$ ) belong to category (ii).

Remarkably, the translocation of a lipid across a membrane closely correlates with the overall reorientation of the lipid measured through the angle between the bilayer normal and the vector directed from the CM of a lipid's hydrocarbon chains to the CM of its head groups (see Figure 2 (Bottom)). When a lipid is accommodated in a membrane leaflet, this tail-to-head vector makes an average angle of $22^{\circ}$ (or $158^{\circ}$ depending on the leaflet) with the bilayer normal. During flip-flop, a lipid changes its orientation, with the corresponding angle lying between the two values being typical for opposite leaflets. Close to the center of the membrane, lipids tend to adopt an orientation perpendicular to the bilayer normal, which is most clearly seen in Figure 2 for a lipid shown by the green curve. It is also noteworthy that lipids' tail-to-head orientation is subject to much larger fluctuations when a lipid is in the middle of a membrane compared to the situation where a lipid is localized in a leaflet; this is due to the fact that lipids in leaflets are more densely packed than lipids in the membrane interior.

\section{Discussion}

Overall, we observed 50 spontaneous flip-flop events (see Table 1). The fact that needs to be emphasized here is that these flip-flop events were coupled to the spontaneous formation of a water pore discussed in refs 28 and 22. For comparison, in numerous atomistic simulation studies of related intact singlecomponent lipid bilayers, we have not identified any flip-flops, despite the long simulation time scales of several microseconds (data not shown). This highlights the importance of water pore formation as a key step in lipid flip-flop.

The flip-flop mechanism observed here takes place in two stages. First, starting from conditions that model the situation in the vicinity of the plasma membrane, our model system includes an initial ionic concentration imbalance across the membrane. Such a transmembrane ion concentration difference is an inherent feature of plasma membranes of eukaryotic cells. ${ }^{31}$ Local fluctuations in ion densities then give rise to a strong local electric field across the bilayer, which in turn initiates the formation of a water pore 22,28 coupled to the subsequent rapid ion leakage that dramatically reduces the local field strength and hence the pore size, yet the pore remains open for a considerably long period of time, which ranges from about $\Delta t$ $=35 \mathrm{~ns}$ to several hundred nanoseconds. We stress that, in the present case, we employed a rather large ion concentration imbalance to promote very fast pore formation. Alternatively, the formation of a water pore could be induced by an external electric field, ${ }^{32-34}$ mechanical stress, ${ }^{32,35}$ or thermal fluctuations. ${ }^{36,37}$ Second, at the same time, the lipids in the bilayer diffuse laterally in the bilayer plane over a distance of $l_{\mathrm{D}}=$ $\sqrt{4 D \Delta t}$, where $D \approx 1 \times 10^{-7} \mathrm{~cm}^{2} / \mathrm{s}$ is a typical lateral diffusion coefficient in fluid lipid bilayers. For a typical lifetime of the pore, $\Delta t \approx 100 \mathrm{~ns}$, the diffusion length of a lipid in the plane of the membrane is $l_{\mathrm{D}} \approx 2 \mathrm{~nm}$, that is, about 3 times the size of the lipid molecule. This implies that there are about a few tens of lipids near the pore that could access it via lateral diffusion before pore closure. Once they do so, many of them undergo flip-flop by translocating through the pore.

Although the average duration of lipid flip-flops through a preformed pore was found to be around $60 \mathrm{~ns}$, flip-flop times for individual lipids scatter considerably and range from 10 to 130 ns. Among factors that influence the rate of lipid translocation, there is reason to mention the significance of pore opening and membrane resealing. Formation of a pore implies
O ns

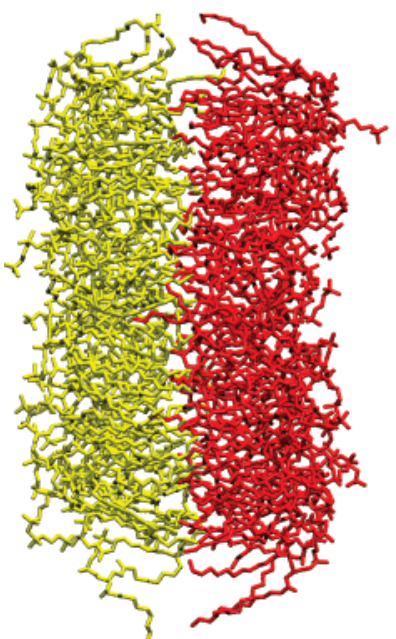

$200 \mathrm{~ns}$

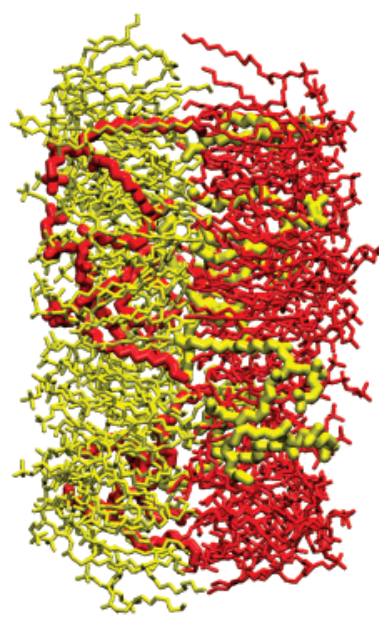

Figure 3. A membrane with the maximal number of lipid flip-flops observed (system 7 in Table 1): intact membrane at $t=0$ ns (left) and membrane with an opened pore at $t=200 \mathrm{~ns}$ (right). Lipids in opposite leaflets are shown in yellow and red; for clarity's sake, the size of flip-flopped lipids has been enlarged.

fast reorientation of some lipid head groups toward the membrane interior, ${ }^{28,32}$ so that the lipids participating in pore formation are moved out of their equilibrium state. If desorption of lipid chains occurs simultaneously with the entering of head groups in the pore, one can observe a very fast lipid flip-flop. In most cases, however, the above scenario takes place only occasionally, since, while a lipid could translocate through half of the membrane, the time required for lipid accommodation in the opposite leaflet can be considerable (see Figure 2). Pore closure, in turn, is able to considerably speed up the flip-flops of partly translocated lipids: Irreversible membrane resealing makes it impossible for such lipids to move back to their original leaflet and effectively pushes them to the opposite one (see, e.g., the translocation of a lipid shown by the red curve in Figure 2 , in which case pore closure occurs at $t \simeq 210 \mathrm{~ns}$ ). Therefore, early pore closure can decrease the duration of flip-flop significantly, as seen for simulation systems 4 and 6 , which have the shortest pore lifetimes (Table 1).

The findings also suggest that pore-mediated translocation of one lipid can promote the flip-flops of others. If a lipid lining a pore translocates to the opposite side of a membrane, it has to be substituted by another lipid from the same leaflet to keep the water pore stable. If this "new" lipid also translocates successfully, the process of substitution needs to be repeated. Indeed, for bilayer system 7 with $\mathrm{KCl}$ salt (Table 1), we observed a sequence of almost concerted flip-flops of three lipids. Remarkably, this particular system is characterized by the largest number of flip-flop events (13 flip-flops in this particular system), which leads to a considerable mixing of lipids from the opposite membrane leaflets over the time span of 200 ns (Figure 3).

The role of salt type was elucidated by considering pores induced by an imbalance of either sodium or potassium cations. In the case of $\mathrm{NaCl}$ salt, one finds 18 successful lipid flip-flops with an average duration of $64 \pm 7 \mathrm{~ns}$. In turn, for systems with $\mathrm{KCl}, 32$ pore-mediated lipid translocations were found to occur with an average duration of $60 \pm 6 \mathrm{~ns}$. Hence, the average times required for lipid flip-flops turn out to be very similar in both cases. However, the type of salt used does affect the probability of lipid flip-flop in the system: bilayers with $\mathrm{NaCl}$ 
and $\mathrm{KCl}$ salt were simulated for 485 and $515 \mathrm{~ns}$, respectively, but the overall number of lipid flip-flops was almost twice as large in the case of $\mathrm{KCl}$. Thus, the probability for a spontaneous flip-flop event to occur is higher by a factor of 1.7 in a bilayer system with potassium cations. This finding correlates well with the fact that sodium ions demonstrate much stronger interactions with zwitterionic phosphatidylcholine lipids than do potassium ions. ${ }^{22}$ In particular, $\mathrm{Na}^{+}$ions are known to bind to carbonyl oxygens of lipids, leading to the formation of tight complexes between neighboring lipid molecules..$^{22,30,38}$ The formation of such complexes most likely hinders considerably the desorption of lipid chains out of the leaflets toward the membrane interior, whereby decreasing the probability of flip-flops.

What, then, is the force that drives lipids residing close to the pore to flip-flop through it? Is it due to the electrostatic coupling between the dipolar head group and the spatially varying electric field (dielectrophoresis), or related to the spontaneous sealing of the hydrophobic membrane region through hydrophobic interactions, or is it simply due to thermal fluctuations? First, let us mention that the lipids considered in this study are neutral, thus the electrostatic potential is not expected to play the main role. We have confirmed this by considering the membrane-normal component of the total electrostatic force exerted on the lipids involved in flip-flops. That is found to fluctuate around zero, without component to drive translocation. Second, however, the spatially inhomogeneous electric field defined by the instantaneous positions of ions could contribute to lipid flip-flops via dielectrophoretic motion, especially at the early stages of pore formation before ion leakage through a pore reduces the local field strength. Therefore, one can expect that the rapid flip-flops observed in the beginning of the simulations could in part be driven by the spatially varying transmembrane electric field due to ion imbalance. Indeed, the contribution of the dielectrophoretic force exerted on the dipole of a lipid at such a high ionic charge imbalance was found to be comparable with thermal fluctuations. However, the flip-flops taking place right after pore formation (which constitute only $20 \%$ out of all flip-flop events) can be considered as being artificially promoted since they are coupled to the high ionic charge imbalance employed in this study, that is, to the method used to create water pores. Furthermore, at longer times, after around 10-20 ns from the moment of pore formation, the transmembrane electric field is almost fully discharged by ion leakage. Under these conditions, the dielectrophoretic force exerted on lipids was found to be about an order of magnitude smaller than the force due to thermal fluctuations. This supports the view that dielectrophoresis is not driving flip-flops. This is particularly true when the system has lost its memory of how the pore was formed. For instance, the ionic charge imbalance for system 7 is essentially zero already at $t=10 \mathrm{~ns}$, implying that lipid flip-flops during the subsequent $190 \mathrm{~ns}$ are hardly affected by the spatially varying local electric field. Third, what we find from the simulations is that the flips and flops are essentially symmetric. The number of flips (events that initiated from the leaflet facing an initial excess of cations) was found to be 22 , while the number of flops (in the opposite direction) was 28. Considering the total number of events, the flip-flops are symmetric within statistical fluctuations.

Thus, one can conclude that the translocation of lipids through transient water pores is mainly diffusive, i.e., driven by thermal fluctuations. Furthermore, very preliminary studies (data not shown) have confirmed that lipid flip-flops can also be observed in bilayer systems where pores are formed by an alternate means without salt ions, lending further support to the above conclusion. Overall, the formation of a water pore spanning the membrane is most likely the only required prerequisite for transmembrane lipid translocation.

The main result of the present study is the observation that the rate of lipid flip-flops is significantly enhanced by the spontaneous formation of water pores. Evidently, one should then ask whether the rate of pore formation is comparable to the lipid flip-flop rate in the absence of proteins or pores. To consider this issue, let us first note that the average time of pore-mediated lipid translocations has here been found to be $\sim 60 \mathrm{~ns}$. For comparison, the flip-flop rate is available from experimental measurements. In particular, on the basis of exchange experiments ${ }^{13}$ performed at $T=323 \mathrm{~K}$ on large unilamellar vesicles composed of DMPC lipids, the upper limit for flip-flop half-time was estimated to be $0.7 \mathrm{~h}$. Assuming that the formation of a water pore in DMPC membranes is the ratelimiting step in the process of flip-flop, we can estimate the probability of pore formation or the pore density $\rho$ in DMPC membranes at $T=323 K$, which is given by ${ }^{16}$ $\rho=\left(t_{\text {flip-flop }} j_{\text {pore }} A_{\text {lipid }}\right)^{-1}$. Here, $t_{\text {flip-flop }}=1 \mathrm{~h}$ is the flip-flop time taken from the experiment, $j_{\text {pore }}=(1 / 60) \mathrm{ns}^{-1}=1.67 \times$ $10^{7} \mathrm{~s}^{-1}$ is the lipid flux through the pore obtained from our MD simulations, and $A_{\text {lipid }}=0.66 \mathrm{~nm}^{2}=0.66 \times 10^{-14} \mathrm{~cm}^{2}$ is the average area per DMPC lipid. This gives us the equilibrium pore density $\rho$ equal to $2.5 \times 10^{3}$ pores $/ \mathrm{cm}^{2}$.

The obtained value for $\rho$ is in reasonable agreement with values available from other sources. In particular, the equilibrium pore density employed in models of electroporation ${ }^{39,40}$ ranges from $0.8 \times 10^{2}$ to $1.5 \times 10^{5}$ pores $/ \mathrm{cm}^{2}$. Tieleman and Marrink reported the density of $\sim 100$ pores $/ \mathrm{cm}^{2}$ for a dipalmitoylphosphatidylcholine (DPPC) bilayer. $^{16}$ Moving on, from the pore density $\rho$ we can estimate the free energy $\Delta G_{\text {pore }}$ required for pore formation; $\Delta G_{\text {pore }}$ is coupled to $\rho$ as ${ }^{16} \rho=\exp \left(-\Delta G_{\text {pore }} / k_{\mathrm{B}} T\right) / A_{\text {lipid. }}$. With $\rho=2.5 \times 10^{3} \mathrm{~cm}^{-2}$ and $T=323 \mathrm{~K}$, one has $\Delta G_{\text {pore }} \simeq 67 \mathrm{~kJ} / \mathrm{mol}$. This value is somewhat smaller than the value of $80 \mathrm{~kJ} / \mathrm{mol}$ reported recently for a DPPC membrane at the same temperature. This difference is not, however, surprising since chains of DMPCs are two hydrocarbons shorter than those of DPPC. This means that a DMPC membrane has a smaller hydrophobic core compared to a DPPC bilayer, and therefore less energy is required for the formation of a pore in the DMPC membrane.

Summarizing, our atomic-scale MD simulations demonstrate that the appearance of a water pore spanning a phospholipid membrane inevitably leads to diffusive transmembrane translocation of lipids through the pore; this translocation occurs spontaneously on a time scale of about $60 \mathrm{~ns}$ and is mainly driven by thermal fluctuations. This strongly supports the idea that the formation of a water pore in a membrane is the ratelimiting step in lipid flip-flop, which typically takes hours. Once a pore has been formed, the subsequent actual pore-mediated lipid translocation occurs extremely fast. Combining our simulation results and available experimental data, we found that the free energy required for pore formation in DMPC membranes at $T=323 \mathrm{~K}$ is about $67 \mathrm{~kJ} / \mathrm{mol}$, in line with previously reported studies. Furthermore, while pore formation can be induced through various ways using, for example, an external electric field, our results show that the formation of pores and the subsequent flip-flops can take place under physiological conditions because of salt ions in the vicinity of the membrane.

In addition to their biological relevance, our findings also offer a molecular-level framework for the development of ways to promote lipid transmembrane translocation and make it 
possible to manipulate lipid distribution across cell membranes. As the present results indicate that the formation of water pores is the only prerequisite for lipid flip-flops, novel techniques should introduce transient defects (pores) into the membranes. In particular, our study provides a molecular-level explanation for the experimentally observed fact that the exposure of lipid membranes to electric field pulses considerably reduces the time required for lipid flip-flops: ${ }^{41}$ External electric field induces electroporation of a membrane, drastically increasing the number of pathways (pores) available for pore-mediated lipid flip-flops. Similarly, the presented pore-mediated mechanism for lipid flipflops can also be invoked to better understand lipid transmembrane asymmetry and to develop means to alter that: by forming defects in membranes, at least local anesthetics, amphiphilic compounds, and pore-forming peptides are expected to facilitate the occurrence of flip-flops. ${ }^{10}$ The view and mechanism presented here is particularly interesting in terms of providing insight into the action of antibacterial agents, which are known as pore-forming peptides and killers of cells. ${ }^{42}$

Overall, the present results provide strong support for previous suggestions ${ }^{16-20}$ and put forward a new mechanism for lipid flip-flop in protein-free membranes under physiological-like conditions, thus updating the present view. It would be of particular interest to elaborate on this matter further by considering how flip-flops take place in asymmetric lipid membranes ${ }^{43}$ characterized by a nonzero membrane potential, which likely plays a role in pore formation together with an ion concentration imbalance across the membrane. That would also clarify the specific role of flippases in plasma membrane-type membranes rich in phosphatidylserines, phosphatidylcholines, and cholesterol.

Acknowledgment. Funding from the Academy of Finland (I.V.) is gratefully acknowledged. The simulations were performed at the Finnish IT Center for Science and on the HorseShoe (DCSC) supercluster at the University of Southern Denmark.

\section{References and Notes}

(1) Gennis, R. B. Biomembranes: Molecular Structure and Function; Springer-Verlag: New York, 1989.

(2) Zachowski, A. Biochem. J. 1993, 294, 1-14.

(3) Manno, S.; Takakuwa, Y.; Mohandas, N. Proc. Natl. Acad. Sci. U.S.A. 2002, 99, 1943.

(4) Pomorski, T.; Hrafnsdottir, S.; Devaux, P. F.; van Meer, G. Semin. Cell Dev. Biol. 2001, 12, 139-148.

(5) Balasubramanian, K.; Schroit, A. J. Annu. Rev. Physiol. 2003, 65 , $701-734$.

(6) Bevers, E. M.; Comfurius, P.; Dekkers, D. W. C.; Zwaal, R. F. A. Biochim. Biophys. Acta 1999, 1439, 317-330. 2921.

(7) Pomorski, T.; Menon, A. K. Cell. Mol. Life Sci. 2006, 63, 2908-

(8) Raggers, R. J.; Pomorski, T.; Holthuis, J. C. M.; Kälin, N.; van Meer, G. Traffic 2000, 1, 226-234.
(9) Marti, J.; Csajka, F. S. Phys. Rev. E 2004, 69, 061918.

(10) Boon, J. M.; Smith, B. D. Med. Res. Rev. 2002, 22, 251-281.

(11) Holthuis, J. C.; Levine, T. P. Nat. Rev. Mol. Cell Biol. 2005, 6, $209-220$.

(12) Kornberg, R. D.; McConnell, H. M. Biochemistry 1971, 10, 11111120 .

(13) Wimley, W. C.; Thompson, T. E. Biochemistry 1990, 29, 12961303.

(14) Toyoshima, Y.; Thompson, T. E. Biochemistry 1975, 14, 15251531.

(15) Schwarz, S.; Haest, C. W. M.; Deuticke, B. Biochim. Biophys. Acta 1999, 1421, 361-379.

(16) Tieleman, D. P.; Marrink, S.-J. J. Am. Chem. Soc. 2006, 128, 12462-12467.

(17) de Vries, A. H.; Mark, A. E.; Marrink, S. J. J. Am. Chem. Soc. 2004, 126, 4488-4489.

(18) Leontiadou, H.; Mark, A. E.; Marrink, S.-J. J. Am. Chem. Soc. 2006, $128,12156-12161$.

(19) Dickey, A. N.; Faller, R. Biophys. J. 2007, 92, 2366-2376.

(20) Kandasamy, S. K.; Larson, R. G. J. Chem. Phys. 2006, 125, 074901.

(21) Berger, O.; Edholm, O.; Jahnig, F. Biophys. J. 1997, 72, 20022013.

(22) Gurtovenko, A. A.; Vattulainen, I. Biophys. J. 2007, 92, 18781890.

(23) Berendsen, H. J. C.; Postma, J. P. M.; van Gunsteren, W. F.; Hermans, J. In Intermolecular Forces; Pullman, B., Ed.; Reidel: Dordrecht, The Netherlands, 1981; pp 331-342.

(24) Lindahl, E.; Hess, B.; van der Spoel, D. J. Mol. Model. 2001, 7, $306-317$.

(25) Darden, T.; York, D.; Pedersen, L. J. Chem. Phys. 1993, 98, 1008910092

(26) Essman, U.; Perera, L.; Berkowitz, M. L.; T. Darden, H. L.; Pedersen, L. G. J. Chem. Phys. 1995, 103, 8577-8592.

(27) Berendsen, H. J. C.; Postma, J. P. M.; van Gunsteren, W. F.; DiNola, A.; Haak, J. R. J. Chem. Phys. 1984, 81, 3684-3690.

(28) Gurtovenko, A. A.; Vattulainen, I. J. Am. Chem. Soc. 2005, 127, $17570-17571$

(29) Sachs, J. N.; Crozier, P. S.; Woolf, T. B. J. Chem. Phys. 2004, $121,10847-10851$.

(30) Gurtovenko, A. A. J. Chem. Phys. 2005, 122, 244902.

(31) Alberts, B.; Bray, D.; Lewis, J.; Raff, M.; Roberts, K.; Watson, J. D. Molecular Biology of the Cell, 3rd ed.; Garland Publishing: New York, 1994.

(32) Tieleman, D. P.; Leotiadou, H.; Mark, A. E.; Marrink, S.-J. J. Am. Chem. Soc. 2003, 125, 6382-6383.

(33) Tieleman, D. P. BMC Biochem. 2004, 5, 10.

(34) Vernier, P. T.; Ziegler, M. J.; Sun, Y.; Chang, W. V.; Gundersen, M. A.; Tieleman, D. P. J. Am. Chem. Soc. 2006, 128, 6288-6289.

(35) Leontiadou, H.; Mark, A. E.; Marrink, S.-J. Biophys. J. 2004, 86, $2156-2164$

(36) Deamer, D. W.; Bramhall, J. Chem. Phys. Lipids 1986, 40, 167188.

(37) Paula, S.; Volkov, A. G.; VanHoek, A. N.; Haines, T. H.; Deamer, D. W. Biophys. J. 1996, 70, 339-348.

(38) Böckmann, R. A.; Hac, A.; Heimburg, T.; Grubmüller, H. Biophys. J. 2003, 85, 1647-1655.

(39) Gowrishankar, T. R.; Esser, A. T.; Vasilkoski, Z.; Smith, K. C.; Weaver, J. C. Biochem. Biophys. Res. Commun. 2006, 341, 1266-1276. (40) Smith, K. C.; Neu, J. C.; Krassowska, W. Biophys. J. 2004, 86, 2813-2826.

(41) Dressler, V.; Schwister, K.; Haest, C. W. M.; Deuticke, B. Biochim. Biophys. Acta 1983, 732, 304-307.

(42) Brogden, K. A. Nat. Rev. Microbiol. 2005, 3, 238-250.

(43) Gurtovenko, A. A.; Vattulainen, I. J. Am. Chem. Soc. 2007, 129, $5358-5359$ 